Atualização rápida

\title{
Prevenção de depressão pós-AVC
}

Prevention of poststroke depression

Escitalopram and problem-solving therapy for prevention of poststroke depression: a randomized controlled trial.
JAMA. 2008;299(20):2391-400.

Bruno Pinatti Ferreira de Souzaㄹ, Marco Antonio Abud Torquato Junior ${ }^{1}$, Simone Maria de Santa Rita Soares²

1 Médico-residente do Departamento de Psiquiatria da Faculdade de Medicina da Universidade de São Paulo (FMUSP).

${ }_{2}^{2}$ Médica preceptora do Departamento e Instituto de Psiquiatria do Hospital das Clínicas da FMUSP.

Recebido: 29/7/2008 - Aceito: 30/7/2008

Souza BPF, et al. / Rev Psiq Clín. 2010;37(4):182

Após um acidente vascular cerebral (AVC), os pacientes podem apresentar uma limitação significativa do desempenho funcional, com repercussões nas relações pessoais, sociais e, sobretudo, na qualidade de vida. No entanto, essas limitações nem sempre se devem ao déficit neurológico, podendo ser causadas por complicações psiquiátricas. No primeiro ano após o AVC, considerado o período agudo, a depressão é a complicação psiquiátrica mais prevalente e a que tem sido mais associada a um pior prognóstico. Os pacientes com essa complicação apresentam recuperação funcional mais lenta, comprometimento das atividades da vida diária, internação hospitalar mais longa na fase aguda e maior mortalidade.

Atualmente, a incidência anual de AVCs nos Estados Unidos supera 700 mil casos e estima-se que $37 \%$ dos pacientes que sobrevivem a um AVC desenvolvem depressão no período agudo. Vários estudos demonstraram a eficácia terapêutica de antidepressivos como nortriptilina, fluoxetina e citalopram no tratamento de depressão pós-AVC. No entanto, sabe-se que apenas $20 \%$ a $50 \%$ dos pacientes com depressão pós-AVC são diagnosticados por médicos não psiquiatras (Schubert et al.) ${ }^{1}$.

Por se tratar de uma complicação prevalente, subdiagnosticada, com grande ônus financeiro e sofrimento humano, atualmente se têm buscado meios para prevenir a depressão pós-AVC. Nesse contexto, Palomäki et al. ${ }^{2}$ utilizaram mianserina, não obtendo resultados significativos, assim como Almeida et al. ${ }^{3}$, que utilizaram sertralina. Rasmussen et al. ${ }^{4}$ demonstraram que a sertralina apresentaria eficácia na prevenção da depressão pós-AVC, mas, ao final do estudo, sua amostra era muito pequena.

Nesse contexto, em 2008 Robinson et al. ${ }^{5}$ realizaram um ensaio clínico randomizado e multicêntrico para avaliar a eficácia de escitalopram ou Terapia Baseada na Solução de Problemas (Problem-Solving Therapy, PST) em comparação ao placebo na prevenção de depressão pós-AVC. Os autores acompanharam 176 pacientes que sofreram AVC isquêmico ou hemorrágico, com idade entre 50 e 90 anos, por um período de 12 meses. Os critérios de exclusão foram depressão na época em que ocorreu o AVC, pacientes com doenças clínicas graves e pacientes que sofreram AVC secundário a malformações intracranianas. Os pacientes foram randomizados em três grupos que receberam escitalopram (59), PST (59) e placebo (58). Os grupos foram comparáveis, à exceção de um maior número de pacientes diabéticos no grupo que recebeu escitalopram. O desfecho principal foi a incidência de depressão maior ou menor de acordo com o DSM-IV. Foram utilizados como desfechos secundários a condição socioeconômica, o índice de atividades da vida diária, o funcionamento social, a avaliação neuropsicológica e os eventos adversos. Os participantes foram avaliados a cada três meses durante um período de um ano. Os grupos de escitalopram e placebo foram duplo-cegos, mas não o de PST.
Os pacientes que receberam placebo foram 4,5 vezes mais propensos a desenvolver depressão do que os pacientes que receberam escitalopram $(\mathrm{p}<, 001)$ e 2,2 vezes mais propensos do que os pacientes que receberam PST $(\mathrm{p}<, 001)$. Além disso, os autores calcularam que, para a prevenção de 1 caso de depressão nessa população, é necessário tratar 7,2 pacientes com escitalopram. Apesar de esse estudo ter resultados significativos para prevenção de depressão pós-AVC, não houve alterações significativas para as variáveis secundárias, o que poderia levar ao questionamento sobre o benefício do tratamento. No entanto, seria precipitado tirar tal conclusão já que o estudo durou apenas um ano, e tais benefícios podem ser evidentes em um prazo mais longo.

Outro dado importante trazido por Robinson et al. 5 é que não foi encontrada diferença significativa entre os grupos quanto à frequência dos efeitos colaterais, o que demonstra ser benéfico tratar esses pacientes de maneira profilática, evitando um maior agravo que uma depressão pode ocasionar.

Conclui-se, portanto, que, apesar de alguns resultados positivos

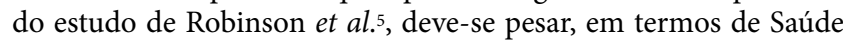
Pública, o custo-benefício do tratamento profilático de depressão pós-AVC. Julgamos que, embora promissores, os dados acima devam ser considerados preliminares e mais estudos com diferentes amostras populacionais e com maior período de acompanhamento se fazem necessários antes que possamos recomendar a profilaxia medicamentosa ou psicoterápica de forma generalizada para a depressão pós-AVC.

\section{Referências}

1. Schubert DSP, Burns R, Paras W, Sioson E. Increase of medical hospital length of stay buy depression in stroke and amputation patients: a pilot study. Psychother Psychosom. 1992;57(1-2):61-6.

2. Palomäki H, Kaste M, Berg A, Lönnqvist R, Lehttihalmes M, Hares J. Prevention of poststroke depression: 1 year randomised placebo controlled double blind trial of mianserin with 6 month follow up after therapy. J Neurol Neurosurg Psychiatry. 1999;66(4):490-4.

3. Almeida OP, Waterreus A, Hankey GJ. Preventing depression after stroke: results from a randomized placebo-controlled trial. J Clin Psychiatry. 2006;67(7):1104-9.

4. Rasmussen A, Lunde M, Poulsen DL, Sorensen K, Qvitzau S, Bech P. A double-blind, placebo-controlled study of sertraline in the prevention of depression in stroke patients. Psychosomatics. 2003;44(3):216-21.

5. Robinson RG, Jorge RE, Moser DJ, Acion L, Solodkin A, Small SL, et al. Escitalopram and problem-solving therapy for prevention of poststroke depression: a randomized controlled trial. JAMA. 2008;299(20):2391-400. 